\title{
Deficiency of $\mathrm{NG2}^{+}$Cells Contributes to the Susceptibility of Stroke-Prone Spontaneously Hypertensive Rats
}

\author{
Pei Wang, Wei-Wei Tian, Jie Song, Yun-Feng Guan \& Chao-Yu Miao \\ Department of Pharmacology, Second Military Medical University, Shanghai, China
}

\author{
Keywords \\ Demyelination; NG2 ${ }^{+}$cells; Oligodendrocyte; \\ SHR-SP; Stroke.

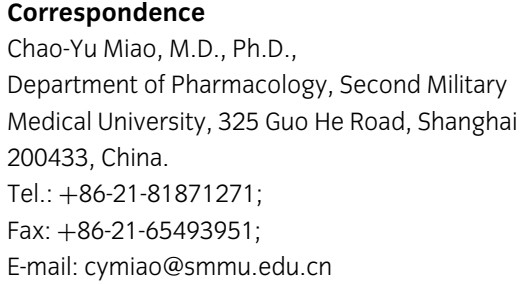

doi: 10.1111/j.1755-5949.2011.00265.x

Pei Wang and Wei-Wei Tian contributed equally to this work.

\begin{abstract}
SUMMARY
Aims: The purpose of this study is to investigate whether the $\mathrm{NG}^{+}$cells, a class of oligodendrocyte progenitor cells, is involved in the pathophysiology of stroke in stroke-prone spontaneously hypertensive rat (SHR-SP). Methods: SHR-SP, SHR, Wistar-Kyoto rats (WKY), and C57BJ/6 mice were used. Immunohistochemistry was conducted to evaluate the number of $\mathrm{NG}^{+}$cells in frozen brain sections. Demyelination was evaluated by Sudan black staining and serum level of myelin basic protein. Middle cerebral artery occlusion (MCAO) was performed to prepare experimental stroke model. Results: The number of $\mathrm{NG}^{+}$cells was significantly decreased in infarct core and increased in penumbra in WKY rats after MCAO. In brain sections of 6-month-old SHR-SP, the number of NG2 $2^{+}$cells was significantly $(P<$ $0.01)$ less than that in age-matched SHR and WKY rats. However, this phenomenon was not observed in 3-month-old rats. Demyelination was found in 6-month-old SHR-SP but not in 3-month-old SHR-SP. Pharmacological treatment of cuprizone in mice induced demyelination and enlargement of cerebral infarction after MCAO. Conclusion: The decline of $\mathrm{NG}^{+}$cells may cause demyelination and contribute to the susceptibility of SHR-SP to ischemic brain injury.
\end{abstract}

\section{Introduction}

Stroke is a leading cause of morbidity and mortality worldwide [1], including China [2-4]. Many efforts for developing effective therapeutic strategies have been made for a long time, whereas the perspective of stoke treatment remains a critical challenge. $\mathrm{NG}^{+}$cells, which are defined by their expression of the chondroitin sulfate proteoglycan NG2, were only deemed as potential oligodendrocyte progenitor cells previously $[5,6]$. In recent years, $\mathrm{NG}^{+}$cells have been proposed to be multipotent neural stem cells [7]. It has been reported that $\mathrm{NG2}^{+}$cells can convert to Schwann cells and possibly astrocytes to repair damage postischemic stroke [7]. $\mathrm{NG2}^{+}$cells were reported to be increased in the penumbra area during stroke and play important roles in neurogenesis after cerebral ischemia [8]. Some researchers found that the majority of newly anagenetic neuronal cells in cortical, penumbra, and contralateral regions were identified as $\mathrm{NG}^{+}$polydendrocytes [9]. Moreover, the neuroprotective characteristic of $\mathrm{NG}^{+}$cells has been revealed recently [10]. However, all the abovementioned work focused on the roles of $\mathrm{NG}^{+}$cells after cerebral ischemia. Whether $\mathrm{NG}^{+}$cells can affect occurrence of stroke remains an unknown issue.

Spontaneously hypertensive rat (SHR) is a well-known inbred strain of genetic hypertension [11], whereas stroke-prone sponta- neously hypertensive rat (SHR-SP) is a substrain of SHR and has been generally accepted as the best animal model for cerebrovascular disease resembling human stroke [12]. Wistar-Kyoto rats represent normal control of SHR and SHR-SP. In SHR-SP, stroke occurs in the course of the development of severe hypertension [12]. In contrast, in SHR, although the hypertension also exists, the stroke is infrequent [12]. Therefore, although the severe hypertension was thought to be one of the most important causes of stroke in SHR-SP, the other causes of stroke in SHR-SP are largely unknown.

We hypothesized that the $\mathrm{NG}^{+}$cells play a role in the occurrence of stroke and tested this hypothesis in the SHR-SP. Moreover, we explored the involvement of demyelination in the association between $\mathrm{NG}^{+}{ }^{+}$cells and ischemic stroke.

\section{Methods}

\section{Animals}

Male SHR-SP, SHR, WKY rats, and C57BJ/6 mice were provided by the Animal Center of our university. All experiments in animals were conducted according to the protocols approved by the Institutional Animal Care and Use Committee at the Second Military Medical University of China. 
A

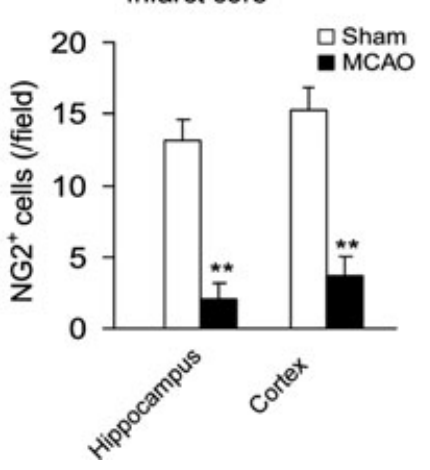

B

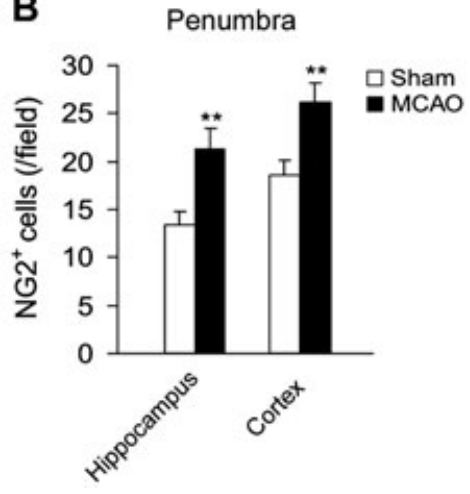

C Unaffected contralateral brain

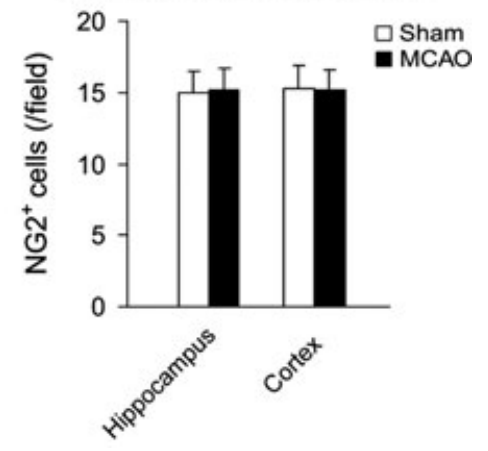

Figure 1 Changes of NG2+ cell number in infarct core (A), penumbra (B), and unaffected contralateral area $(\mathbf{C})$ in WKY rats undergoing MCAO operation. $\mathrm{n}=6$ per group. ${ }^{* *} P<0.01$ versus sham operation.

\section{Determination of Brain NG2 ${ }^{+}$Cells}

$\mathrm{NG}^{+}$cells were detected with immunohistochemistry as described previously $[13,14]$. In brief, frozen $20-\mu \mathrm{m}$-thick brain sections were fixed in $4 \%$ paraformaldehyde, blocked by $8 \%$ normal goat serum and incubated in specific primary antibody $\left(\mathrm{NG}^{+}\right.$; 1:200; Chemicon, CA, USA) and Cy3-conjugated secondary antibody in sequence. Images were obtained by fluorescence microscope (IX-71; Olympus, Tokyo, Japan) and analyzed using ImagePro Plus software [Media Cybernetics, Silver Spring, MD, USA; Ref.15]. The number of $\mathrm{NG}^{+}$cells in hippocampus and cortex was evaluated. For each animal, at least five brain sections and at least five different areas in one section were evaluated.

\section{Sudan Black Staining}

The Sudan black staining technique was used to detect the demyelination lesion. Frozen sections were left at room temperature, rinsed with 0.01M Phosphate Buffered Saline (PBS), and stained with Sudan black in $70 \%$ ethanol solution for $30 \mathrm{~min}$ [16]. The specimens were differentiated in $50 \%$ ethanol, rinsed with distilled water, and mounted in a mixture of glycerol/PBS.

\section{Enzyme-linked Immunosorbent Assay (ELISA)}

Demyelination was also evaluated by detecting serum level of myelin basic protein (MBP) with a commercial ELISA kit (Uscnlife, Missouri, TX, USA). In brief, the blood of mice was obtained from tail [17]. Serum was incubated at room temperature with sample buffer in 96-well plates for $90 \mathrm{~min}$ and then with biotin-labeled anti-MBP detection antibody for $60 \mathrm{~min}$. Finally, a streptavidin-horseradish peroxidase conjugate was added at room temperature for $30 \mathrm{~min}$. Bound MBP was detected by adding tetramethylbenzidine substrate solution for $15 \mathrm{~min}$ and the plates were read at $450 \mathrm{~nm}$ as described previously [18].

\section{Middle Cerebral Artery Occlusion (MCAO)}

MCAO was performed to prepare an experimental stroke model according to standard protocol [13]. The core temperature (rectum) was maintained at $36.5-37.5^{\circ} \mathrm{C}$ by a homeothermic heating pad. Cerebral focal ischemia was produced by intraluminal occlusion of the left middle cerebral artery, using a silicone rubbercoated nylon monofilament [19]. Cortical blood flow was measured with a laser Doppler flowmeter (Moor Instruments, Axminster, UK) to ensure cerebral blood flow was reduced by more than $85 \%$ [20]. Two hours after MCAO, the occluding filament was withdrawn to allow reperfusion.

\section{Statistical Analysis}

Data are expressed as mean \pm SEM. Differences were evaluated by two-tailed Student's $t$-test (two groups) or one-way analysis of variance followed by SNK post hoc test (three or more groups). Statistical significance was set at $P<0.05$.

\section{Results}

\section{The Number of NG2 ${ }^{+}$Cells Changes After Ischemic Stroke in Normal Control Rats}

We first determined the change of brain $\mathrm{NG}^{+}$cells after ischemic stroke in WKY rats, which are normal control rats of SHR-SP. As shown in Figure 1(A), in the infarct core of MCAO rats, the number of $\mathrm{NG}^{+}$cells in both hippocampus and cortex was reduced significantly compared with that in sham-operated rats $(P<0.01)$, whereas in the penumbra area of MCAO rats, the number of $\mathrm{NG}^{+}$ cells was increased significantly (Figure 1B; $P<0.01$ ). The number of $\mathrm{NG}^{+}$cells in unaffected contralateral region of MCAO rats was not altered (Figure 1C). 
A

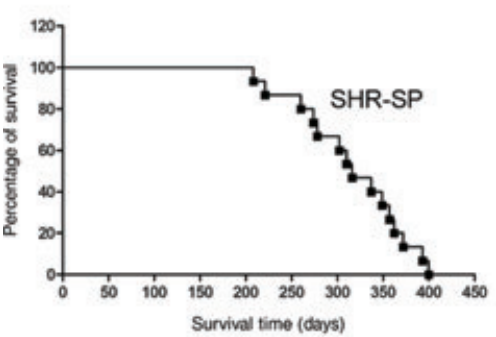

B

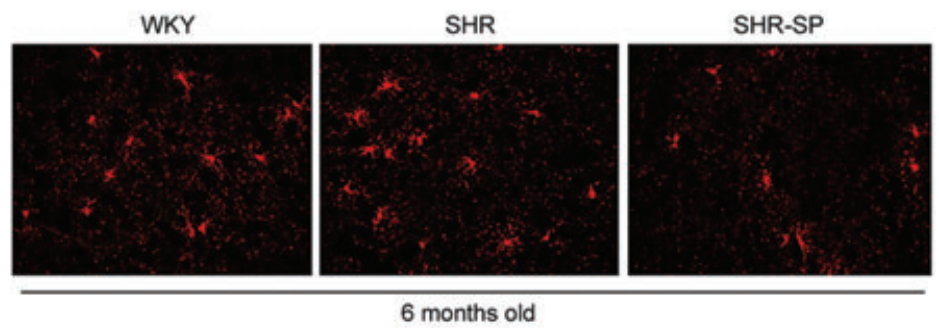

$\mathrm{E}$

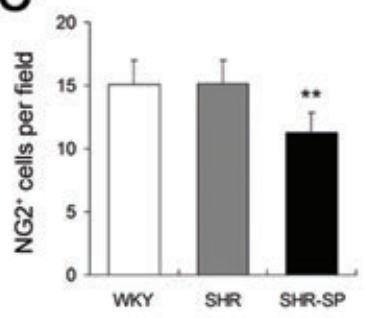

D

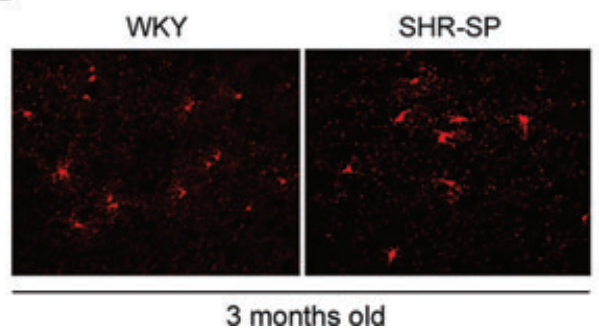

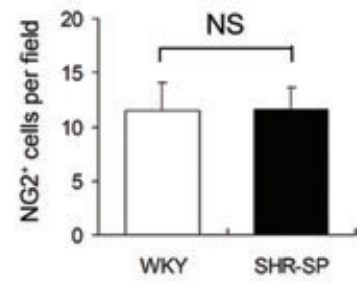

Figure 2 Decline of brain NG2 ${ }^{+}$cells in 6-month-old SHR-SP but not in 3-month-old SHR-SP. (A) Survival curve of SHR-SP. (B,C) Representative image of NG2 ${ }^{+}$ cells in hippocampus and cortex and quantitative analysis of NG2+ cells in 6-month-old SHR-SP, SHR, and WKY rats. $n=6$ per group. ${ }^{* *} P<0.01$ versus WKY (D,E) Representative image of $\mathrm{NG}^{+}$cells and quantitative analysis of $\mathrm{NG}^{+}$cells in 3-month-old SHR-SP. $n=6$ per group. NS, no significance.

\section{The Number of NG2 ${ }^{+}$Cells Decreases in 6-Month-Old SHR-SP but Not in 3-Month-Old SHR-SP}

In this study, we observed that the SHR-SP began to die at about 6 months after birth (Figure 2A). All dead rats were examined for the stroke lesion. This result suggests that the susceptibility to stroke in the SHR-SP emerges at around 6 months old. In 6month-old SHR-SP, the number of $\mathrm{NG}^{+}$cells in hippocampus and cortex was significantly less than that in WKY rats (Figure 2B and C). However, the SHR, another hypertensive rat strain without stroke susceptibility, did not display the similar feature (Figure $2 \mathrm{~B}$ and $\mathrm{C}$ ). In contrast, in 3-month-old SHR-SP, the number of $\mathrm{NG}^{+}$cells was comparable to that in age-matched WKY rats (Figure $2 \mathrm{D}$ and $\mathrm{E}$ ). These data strongly suggest that the decrease of number of $\mathrm{NG}^{+}$cells at 6 months old in SHR-SP is tightly associated with the occurrence of stroke.

\section{Demyelination Lesion in 6-Month-Old SHR-SP}

Because $\mathrm{NG}^{+}$cells are oligodendrocyte progenitor cells and oligodendrocyte is a major component of medullary sheath in central nervous system, we next examined the demyelination in SHRSP without stroke. In 6-month-old SHR-SP, the ratio of optical density in white matter (corpus callosum) was significantly lower than that in WKY rats (Figure 3A and B; $P<0.001$ ). Moreover, serum level of MBP (a marker of demyelination) in 6-month-old SHR-SP was accordingly higher than that in WKY rats (Figure 3C; $P<0.01)$. These phenomena were not observed in 3-month-old
A

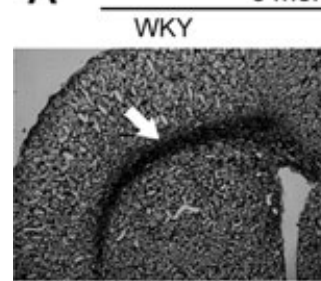
6 months old

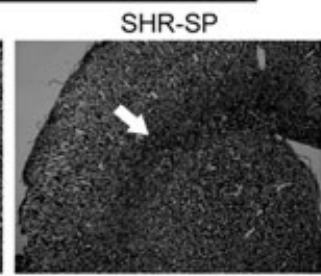

Sudan Black staining
B

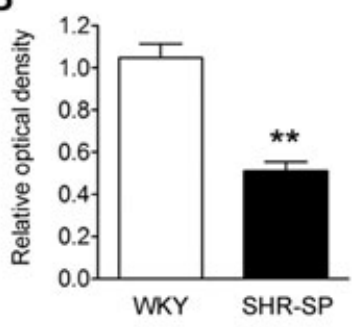

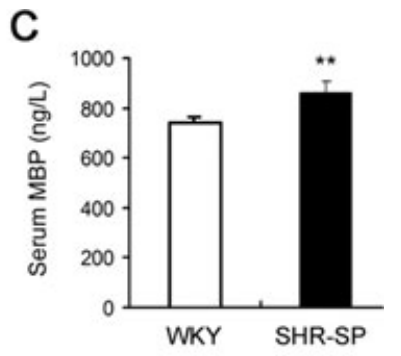

Figure 3 Demyelination in white matter of 6-month-old SHR-SP. (A,B) Representative image (A) and quantitative analysis (B) of Sudan black staining showing demyelination in 6-month-old SHR-SP. (C) Serum MBP level in 6-month-old SHR-SP is higher than that in age-matched WKY rats. $n=6-8$ per group. **P $<0.01$ versus WKY. 
A

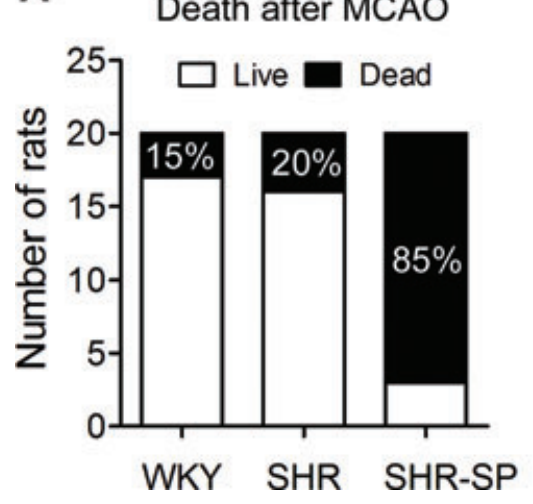

B

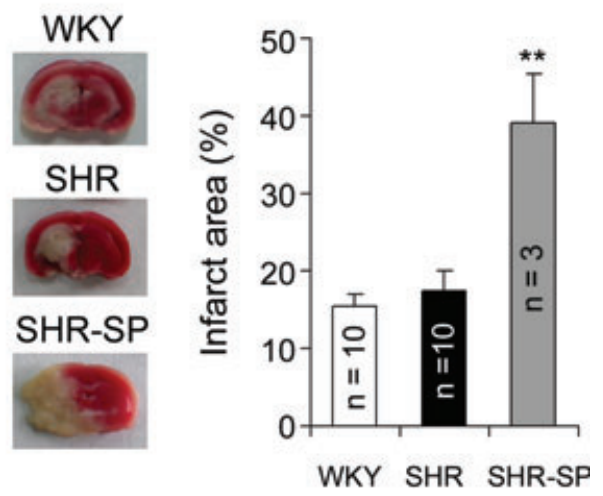

Figure 4 The vulnerability to cerebral ischemic injury differs in WKY, SHR, and SHR-SP. (A) The difference of outcome after MCAO in age-matched WKY, SHR, and SHR-SP. $n=20$ per group. (B) The representative image and quantitative data of the cerebral infarct area in alive rats after $M C A O$. $* * P<01$ versus WKY or SHR.

SHR-SP and WKY rats (data not shown). These data indicate that the progress of demyelination lesion in SHR-SP may result from the decrease of $\mathrm{NG}^{+}$cells.

\section{Vulnerability to Cerebral Ischemic Injury Differs in WKY, SHR, and SHR-SP}

We performed MCAO in 6-month-old WKY, SHR, and SHR-SP. There were significant differences among the outcome at $24 \mathrm{~h}$ after MCAO in three strains. The death rate was $15 \%$ in WKY and $20 \%$ in SHR, whereas was $85 \%$ in SHR-SP (Figure $4 \mathrm{~A}$ ). We examined the infarct area in live rats. The relative infarct area SHR-SP was significantly higher than that in WKY and SHR (Figure 4B).

\section{Demyelination Aggravates the Outcome of Ischemic Stroke}

To evaluate whether demyelination can affect stroke injury, we treated mice with cuprizone (CPZ) for 6 weeks to induce demyeli- nation. The ratio of optical density of corpus callosum in CPZtreated mice was lower than that in control mice (Figure $5 \mathrm{~A}$ and B; $P<0.01)$. The serum MBP levels in CPZ-treated mice were also enhanced significantly (Figure 5C; $P<0.01$ ). CPZ treatment aggravated the cerebral infarction induced by MCAO (Figure 5D and E).

\section{Discussion}

The main findings of this study are that we found the decline of $\mathrm{NG}^{+}$cell number in SHR-SP at 6 months after birth, when the fatal stroke began to appear. The absence of this phenotype in SHR may exclude the possibility that the decline of $\mathrm{NG}^{+}$cells number is because of the hypertension to some extent. The finding that the $\mathrm{NG}^{+}$cell number in 3-month-old SHR-SP is comparable to that in control rats further excludes the decline of $\mathrm{NG}^{+}$cell number in SHR-SP is congenital. Therefore, these results straightforwardly indicate that the decline of $\mathrm{NG}^{+}$cells number in 6-month-old SHR-SP is a unique feature in SHR-SP, and may be tightly associated with the fatal stroke occurrence in SHR-SP.
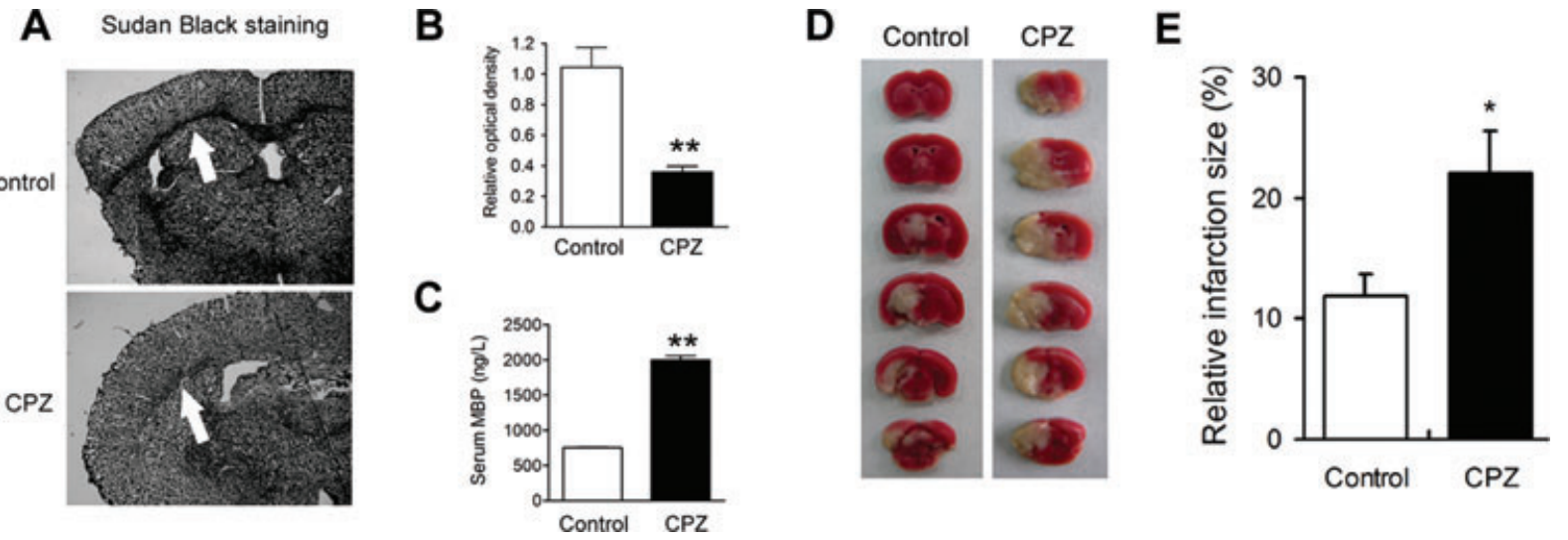

Figure 5 Demyelination induced by cuprizone (CPZ) aggravates brain injury upon MCAO. Sudan black staining (A) and (B) and serum MBP levels (C) evidence the demyelination in CPZ-treated mice. (D,E) Demyelination aggravates brain injury induced by MCAO. $n=6-8$ per group. ${ }^{*} P<0.05 ; * * P<0.01$ versus control. 
Ischemic stroke, the main kind of stroke, is an age-related [20-22] and gene-related [23,24] disease and always followed by long-term hypertension and other cardiovascular diseases such as carotid plaque [25-27], intracranial atherosclerotic lesions, $[28,29]$ and arterial fibrillation [30-32]. Moreover, hyperlipemia, diabetes, smoke, alcohol abuse, obesity, salt intake, and many other factors also contribute to the pathogenesis of stroke $[1,3,4,20,23,33-38]$. After the occlusion of blood supply, severe hypoxia and activation of many toxic metabolites, such as reactive oxygen species, excitatory amino acid and excessive calcium ion, could trigger more deteriorative signal cascades and further damage the brain tissue around the lesions. However, because the effective treatments for stroke are limited yet, prevention of stroke may be a promising way to curb the stroke pandemic $[39,40]$, yielding that detection of early signs of stroke in animal model is very valuable for future clinical studies.

SHR-SP is the one of most suitable models for the stroke study. Many factors, such as cerebral endothelial dysfunction [41,42], blood-brain-barrier impairment [43], and oxidative stress [44], have been previously revealed. It is widely believed that the causes of stroke in SHR-SP are very complex. However, the difference of neuronal cell between SHR-SP and control has never been reported. Thus, when we found the deficiency of $\mathrm{NG}^{+}$cells at the malignant stage in SHR-SP, we proposed that the decline of the number of $\mathrm{NG}^{+}$cells might induce decline of oligodendrocyte and then dysfunction of medullary sheath, which at last lead to the susceptibility to stroke. As expected, in the following studies, marked demyelination was observed in 6-month-old SHR-SP but not in 3-month-old SHR-SP, strongly supporting this assumption. It has been recognized that the oligodendrocytes, myelin-forming glial cells, are vulnerable to damage in cerebral ischemia [45], and there is growing awareness that oligodendrocytes are also therapeutic targets of injury in acute ischemia. However, whether demyelination, which commonly happens in white matter, can aggravate the neuronal injury during acute ischemia is unknown. To test this hypothesis, we studied the effect of demyelination on cerebral infarction, using CPZ feeding [46]. In CPZ-treated mice, the marked enlarged cerebral infarction size by MCAO confirmed our speculation.

In conclusion, our results suggest that the deficiency of $\mathrm{NG}^{+}$ cells may susceptibility of SHR-SP to stroke, and implicate that the patients with demyelination, even slight, may be more vulnerable to cerebral ischemia injury.

\section{Acknowledgments}

This work was supported by grants from the National Basic Research Program of China (2009CB521902 to C.-Y.M.), the National Natural Science Foundation of China (81130061 to C.-Y.M. and 81100866 to P.W.), the Program of Shanghai Subject Chief Scientist (10XD1405300 to C.-Y.M.), the Shanghai "Shu Guang" Project (10GG19 to C-.Y.M.), and the Open Funds of the Shanghai Key Laboratory of Vascular Biology (GXY2009001005 to P.W.).

\section{References}

1. Donnan GA, Fisher M, Macleod M, Davis SM. Stroke. Lancet 2008;371:1612-1623.

2. He J, Gu D, Wu X, et al. Major causes of death among men and women in China. $N$ Engl J Med 2005;353:1124-1134.

3. Bazzano LA, Gu D, Whelton MR, et al. Body mass index and risk of stroke among Chinese men and women. Ann Neurol 2010;67:11-20

4. Kurth T, Leitzmann MF. Awakening of the sleeping giant: obesity and stroke in China. Ann Neurol 2010;67:1-2.

5. Zhu X, Bergles DE, Nishiyama A. NG2 cells generate both oligodendrocytes and gray matter astrocytes. Development 2008; 135:145-157.

6. Belachew S, Chittajallu R, Aguirre AA, et al. Postnatal NG2 proteoglycan-expressing progenitor cells are intrinsically multipotent and generate functional neurons. J Cell Biol 2003;161:169-186.

7. Richardson WD, Young KM, Tripathi RB, McKenzie I. NG2-glia as multipotent neural stem cells: Fact or fantasy? Neuron 2011;70:661-673.

8. Tanaka K, Nogawa S, Ito D, et al. Activation of NG2-positive oligodendrocyte progenitor cells during post-ischemic reperfusion in the rat brain. Neuroreport 2001;12:2169-2174

9. Komitova M, Perfilieva E, Mattsson B, Eriksson PS, Johansson BB. Enriched environment after focal cortical ischemia enhances the generation of astroglia and NG2 positive polydendrocytes in adult rat neocortex. Exp Neurol 2006; 199:113-121.

10. Smirkin A, Matsumoto H, Takahashi $\mathrm{H}$, et al. $\mathrm{Ibal}+/ \mathrm{NG} 2+$ macrophage-like cells expressing a variety of neuroprotective factors ameliorate ischemic damage of the brain. J Cerebr Blood F Met 2011;30:603-615.
11. Miao CY, Xie HH, Zhan LS, Su DF. Blood pressure variability is more important than blood pressure level in determination of end-organ damage in rats. J Hypertens 2006;24:1125-1135.

12. Paschen W, Mies G, Bodsch W, Yamori Y, Hossmann KA. Regional cerebral blood flow, glucose metabolism, protein synthesis, serum protein extravasation, and content of biochemical substrates in stroke-prone spontaneously hypertensive rats. Stroke 1985; 16:841-845.

13. Wang P, Xu TY, Guan YF, et al. Nicotinamide phosphoribosyltransferase protects against ischemic stroke through SIRTl-dependent adenosine monophosphate-activated kinase pathway. Ann Neurol 2011;69:360-374.

14. Speidl WS, Cimmino G, Ibanez B, et al. Recombinant apolipoprotein A-I Milano rapidly reverses aortic valve stenosis and decreases leaflet inflammation in an experimental rabbit model. Eur Heart J 2010;31:2049-2057.

15. Harrington EP, Zhao C, Fancy SPJ, Kaing S, Franklin RJM, Rowitch DH. Oligodendrocyte PTEN is required for myelin and axonal integrity, not remyelination. Ann Neurol 2010;68:703-716.

16. Voermans NC, van Alfen N, Pillen S, et al. Neuromuscular involvement in various types of Ehlers-Danlos syndrome. Ann Neurol 2009;65:687-697.

17. Wang P, Yang FJ, Du H, et al. Involvement of leptin receptor (LepRb)-STAT3 signaling pathway in brain FTO downregulation during energy restriction. Mol Med 2011;17:523-532.

18. Venteo L, Bourlet T, Renois F, et al. Enterovirus-related activation of the cardiomyocyte mitochondrial apoptotic pathway in patients with acute myocarditis. Eur Heart $J$ 2010;31:728-736.

19. Nyakas C, Felszeghy K, Szabó R, et al. Neuroprotective effects of vinpocetine and its major metabolite cis-apovincaminic acid on nmda-induced neurotoxicity in a rat entorhinal cortex lesion model. CNS Neuroscience $\theta$ Therapeutics 2009;15:89-99.

20. Arumugam TV, Phillips TM, Cheng A, Morrell CH, Mattson MP, Wan R. Age and energy intake interact to modify cell stress pathways and stroke outcome. Ann Neurol 2010;67:41-52.

21. Yager JY, Thornhill JA. The effect of age on susceptibility to hypoxic-ischemic brain damage. Neurosci Biobehav Rev 1997;21:167-174.

22. Sánchez MG, Bourque $M$, Morissette $M$, Di Paolo $T$. Steroids-Dopamine Interactions in the Pathophysiology and Treatment of CNS Disorders. CNS Neuroscience $\theta$ Therapeutics 2010; 16:e43-e71.

23. Jickling GC, Xu H, Stamova B, et al. Signatures of cardioembolic and large-vessel ischemic stroke. Ann Neurol 2010;68:681-692.

24. Guo JM, Liu AJ, Su DF. Genetics of stroke. Acta Pharmacol Sin 2010;31:1055-1064

25. Vilela P, Goulao A. Ischemic stroke: Carotid and vertebral artery disease. Eur Radiol 2005; 15:427-433.

26. Matter CM, Stuber M, Nahrendorf M. Imaging of the unstable plaque: How far have we got? Eur Heart $J$ 2009;30:2566-2574

27. Andersen ZJ, Olsen TS, Andersen KK, Loft S, Ketzel M, Raaschou-Nielsen O. Association between short-term exposure to ultrafine particles and hospital admissions for stroke in Copenhagen, Denmark. Eur Heart $J$ 2010;31:2034-2040

28. Qureshi AI, Feldmann E, Gomez CR, et al. Intracranial atherosclerotic disease: An update. Ann Neurol 2009; 66:730-738

29. Klingenberg R, Hansson GK. Treating inflammation in atherosclerotic cardiovascular disease: Emerging therapies Eur Heart J 2009;30:2838-2844. 
30. Friberg L, Hammar N, Rosenqvist M. Stroke in paroxysma atrial fibrillation: Report from the Stockholm Cohort of Atrial Fibrillation. Eur Heart J 2010;31:967-975.

31. Lubitz SA, Rosen AB, Ellinor PT, Benjamin EJ. Stroke risk in AF: Do AF patterns matter? Eur Heart $J$ 2010;31:908-910.

32. Lip GY, Rasmussen LH, Olsson SB, et al. Oral direct thrombin inhibitor AZD0837 for the prevention of stroke and systemic embolism in patients with non-valvular atrial fibrillation: A randomized dose-guiding, safety, and tolerability study of four doses of AZD0837 vs. vitamin K antagonists. Eur Heart J 2009;30:2897-2907.

33. Putaala J, Haapaniemi E, Metso AJ, et al. Recurrent ischemic events in young adults after first-ever ischemic stroke. Ann Neurol 2010;68:661-671.

34. Seok JM, Kim SG, Kim JW, et al. Coagulopathy and embolic signal in cancer patients with ischemic stroke. Ann Neurol 2010;68:213-219.

35. Basu A, Graziadio S, Smith M, Clowry GJ, Cioni G, Eyre JA. Developmental plasticity connects visual cortex to motoneurons after stroke. Ann Neurol 2010;67:132-136.

36. Shiue I. Does body mass index increase risk of hemorrhagic stroke? Ann Neurol 2010;68:115-1 16; author reply 116.

37. Johnston SC, Sidney S, Hills NK, et al. Standardized discharge orders after stroke: Results of the quality improvement in stroke prevention (QUISP) cluster randomized trial. Ann Neurol 2010;67:579-589.

38. Cheung N, Liew G, Lindley RI, et al. Retinal fractals and acute lacunar stroke. Ann Neurol 2010;68:107-111.

39. Putaala J, Haapaniemi E, Metso AJ, et al. Recurrent ischemic events in young adults after first-ever ischemic stroke. Ann Neurol 2010;68:661-671.

40. Liu AJ, Ma XJ, Shen FM, Liu JG, Chen H, Su DF. Arterial baroreflex: A novel target for preventing stroke in rat hypertension. Stroke 2007;38:1916-1923.

41. Ghisdal P, Godfraind T, Morel N. Effect of nitro-L-arginine on electrical and mechanical responses to acetylcholine in the superior mesenteric artery from stroke-prone hypertensive rat. Br J Pharmacol 1999;128:1513-1523.

42. Jesmin S, Sakuma I, Togashi H, et al. Altered expression of endothelin and its receptors in the brain of SHR-SP at malignant hypertensive stage. $J$ Cardiovasc Pharmacol 2004;44 (Suppl 1):S1 1-S15.

43. Lippoldt A, Kniesel U, Liebner S, et al. Structural alterations of tight junctions are associated with loss of polarity in stroke-prone spontaneously hypertensive rat blood-brain barrier endothelial cells. Brain Res 2000;885:251-261.

44. Nagotani S, Hayashi T, Sato K, et al. Reduction of cerebral infarction in stroke-prone spontaneously hypertensive rats by statins associated with amelioration of oxidative stress. Stroke 2005;36:670-672.

45. Dewar D, Underhill SM, Goldberg MP. Oligodendrocytes and ischemic brain injury. $J$ Cereb Blood Flow Metab 2003;23:263-274.

46. Torkildsen O, Brunborg LA, Myhr KM, Bo L. The cuprizone model for demyelination. Acta Neurol Scand Suppl 2008; 188:72-76 